\title{
High Energy Pulsars: Pulses and Populations
}

\author{
Roger W. Romani ${ }^{1}$ \\ Department of Physics, Stanford University, CA 94305-4060 \\ rur@astro.stanford.edu
}

\begin{abstract}
Recent X-ray and $\gamma$-ray observations have detected a number of isolated spin-powered pulsars. Studies of the pulse profiles of these objects are providing a useful guide to the site of the high energy emission. The $\gamma$-ray pulses, which are closely tied to the primary population of radiating particles, seem to be an especially useful discriminant between pulsar models. With an understanding of how the pulsar luminosity and beaming factors evolve with spin parameters, pulsar population syntheses can also be used to check the predictions of high energy emission models. In turn, comparison with $\gamma$-ray sky surveys constrains the properties of the young pulsar population.
\end{abstract}

\section{Introduction}

The ROSAT, ASCA and CGRO missions have been quite successful at identifying young spin-powered pulsars. ROSAT, for example has now seen emission from $\sim 15$ young isolated pulsars (Becker 1996). The EGRET experiment on $C G R O$ has now detected at least 6 pulsars (Thompson, et al.1994, Fierro 1996); while $A S C A$ provides interesting constraints on the $2-10 \mathrm{keV}$ spectra of several pulsars and their surrounding nebulae (Kawai, these proceedings). These observations have produced a number of high quality pulse profiles, and in case of of the brightest EGRET pulsars sufficient signal to noise is available to examine the spectral variations with pulsar phase. In this paper, we describe how these new data are changing our view of pulsar radiation mechanisms and review our current efforts to understand the Galactic population of high energy pulsars.

\section{High Energy Emission Models and Pulse Profiles}

The earliest pulse detections at hard X-ray $/ \gamma$-ray energies were those of the Crab (Fishman, Harnden and Haymes 1969; Hillier et al. 1970) and Vela pulsars (Thompson 1975). Both objects displayed two strong pulses, separated by $\sim 150^{\circ}$ of phase. Even though there was evidence for emission between these pulses, to usual astronomical accuracy $150^{\circ} \approx 180^{\circ}$ and the natural interpretation was that one was viewing opposite magnetic poles. The high energy observational situation did not change much through the COS-B era, with no

${ }^{1}$ Alfred P. Sloan Fellow 

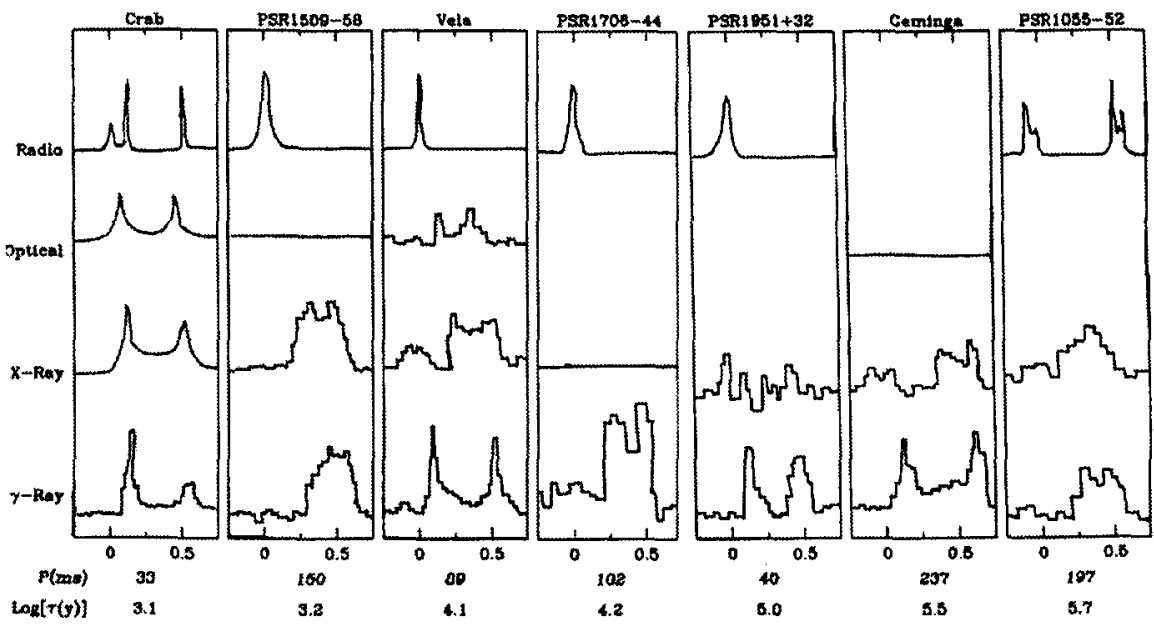

Figure 1. Compilation of pulse profiles for young high energy pulsars. The closest approach of the magnetic axis is at phase 0 . Horizontal lines at a given waveband indicate a counterpart detection, but no known pulse profile; blanks indicate no detections.

further convincing pulse detections. With Einstein, pulsed emission was seen from the Crab-like pulsars PSR1509-58 and PSR0540-69 in the LMC; both of these objects displayed single broad pulses below $\sim 5 \mathrm{keV}$. The soft X-ray pulses were interpreted as thermal emission from heated polar caps and thus did not provide much impetus to refine models of the high energy $\gamma$-ray emission.

The recent generation of space missions has changed this situation significantly. Six pulsars have now been seen above $100 \mathrm{MeV}$ by EGRET and PSR150958 has been detected to $\sim \mathrm{MeV}$ energies. Also, relative phasing of the energy bands is now largely determined. A compilation of pulse profiles is shown in Figure 1. Pulse profiles are plotted with the phase of the closest approach of the magnetic axis, generally estimated from radio pulse and polarization data, at 0 . Two features seem common to the sample of young pulsars: first, the $\mathrm{GeV}$ $\gamma$-ray emission seems to arrive as a double pulse with bridge emission in between or as an unresolved close double/broad single pulse. Second the radio profiles generally bear little resemblance to the high energy pulses (with the exception of the Crab) and seem to lead the energetic emission in pulse phase. In fact for Geminga, rather deep searches have determined no radio pulse whatsoever.

The shape of the pulse profile has driven $\gamma$-ray modeling efforts to move to a single pole picture. An edge-brightened cone of emission associated with a magnetic pole seems the only natural way to generically produce a broad pulse bounded by brighter peaks. Two methods to attain such a profile with widths of $\varsigma 180^{\circ}$ have been pursued in the literature. The first is a descendent of the polar cap model, recently elaborated for curvature-induced pair cascades by Daugherty and Harding (1996, DH) and for Compton scattering-controlled cap acceleration by Sturner, Dermer and Michael $\left(1995\right.$, SDM). To achieve broad $>150^{\circ}$ pulses in these low altitude models, the magnetic axis must be aligned to within roughly 


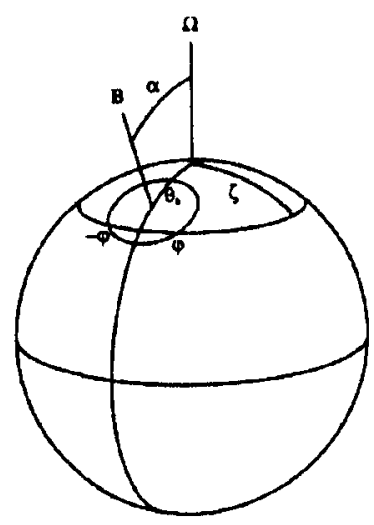

Figure 2. Schematic of the aligned polar cap picture (Daugherty and Harding). The edge-brightened emission from the polar cap ring give pulses at $\pm \phi$. For a wide profile we require $\alpha \lesssim \theta_{b}$.

the polar cap opening angle (Figure 2). In this scheme, outer sight-lines $(\zeta>\alpha)$ produce pulses of width $<180^{\circ}$, while inner sightlines (passing between $B$ and $\Omega$ ) can produce pulses broader than $180^{\circ}$. The second route to an edge-brightened cone of emission is demonstrated in the outer gap model described by Chiang and Romani (1994) and Romani and Yadigaroglu (1995, RY95). This model places the emission near the open zone boundary above the magnetic pole, at altitudes up to several tenths of the light cylinder radius. The emission surface can be roughly pictured as half of a widely flaring, curved hollow cone - the effects of relativistic aberration and time-of-flight across the light cylinder are important in computing the resulting pulse profile (Figure 3). For both models, double pulses are generic and broad single pulses occur when the line of sight sweeps across the edge of the hollow cone.

The results of pulse profile modeling can be compared with the high quality data obtained for the brightest $\mathrm{GeV}$ pulsar, Vela (Figure 4). Here we show the $E G R E T E>100 \mathrm{MeV}$ pulse profile along with the radio pulse and the model light curves of SDM, DH and RY95. In the case of the polar cap models $\alpha$ has been chosen to fit the pulse width with $\alpha \sim \theta_{\text {cap. The DH model allows }}$ acceleration to several stellar radii, while the SDM model invokes a polar cap $\sim 4 \times$ larger than the vacuum dipole value. The pulse profile computed by RY95 is that for a substantially larger inclination $\alpha=65^{\circ}$. For all models the closest approach of the magnetic axis (and under standard assumptions, the radio pulse and maximum of the radio polarization sweep rate) is at phase 0 . Note that there are 'inner' line of sight configurations for the polar caps that give rather similar $\gamma$-ray profiles (peaks inverted), but would have the radio peak at phase 0.5 (dashed lines). While the pulse profile modeling helps pin down the location of the high energy emission, constraints on physical conditions in the emission zone are probably best extracted from pulse spectra. Early results of spectral modeling which show some success at matching phase resolved spectra of Vela can be found in DH and Romani (1996). 


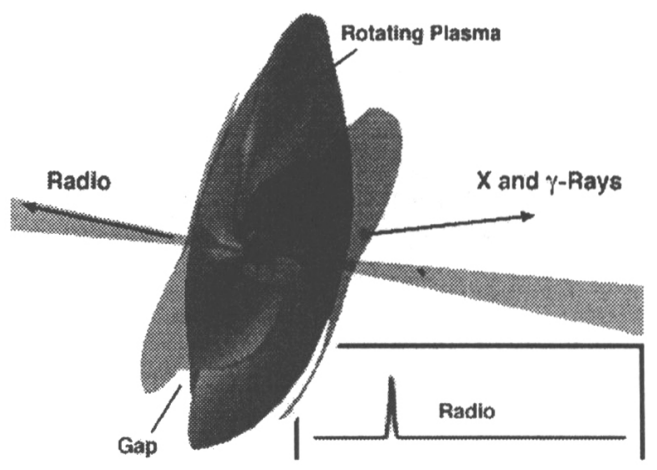

Figure 3. Geometry of the magnetosphere model of RY95. Radio pulses beam along the magnetic axes. Hard X-rays and $\gamma$-rays are emitted from field lines bounding the upper surfaces of the acceleration gaps. The gaps are also bounded by the plasma-filled closed zone.

The ROSAT X-ray pulse detections (sketched for several pulsars in Figure 1) are quite complex. Ögelman and colleagues have made important progress by noting that young $\sim 10^{4}-10^{6} \mathrm{y}$ pulsars often have low energy $(0.1-0.5 \mathrm{keV})$ pulses shifted in phase from a high energy ( $>0.5 \mathrm{keV}$ ) pulse (see Ögelman 1995). The low energy pulse emission (and the bulk of the X-ray flux) seem to be thermal in na.ture. Although the hard pulse appears to connect in phase and in spectrum with the hard X-ray to $\gamma$-ray magnetospheric emission, the interpretation is not yet clear: DH96 suggest that it is a hot polar cap, while RY95 identify it as magnetospheric flux. Sensitive X-ray spectroscopy from $A S^{\prime} C A$ and future missions can help resolve this issue. It seems likely that the complex behavior in the X-ray range will provide rich diagnostics for the high energy emission physics.

\section{Plane Surveys and the Energetic Pulsar Population}

In addition to the pulse properties of individual objects, the distribution of pulsar luminosities and the number of pulsar sources in flux limited surveys provide important constraints on high energy pulsar models. Gamma-ray observations of known pulsars show that they are very bright in the $\mathrm{GeV}$ range, directing as much as several tenths of their spindown power into $\mathrm{GeV}$ photons and dominating the point source component of the $\gamma$-ray sky. Accordingly Galactic plane surveys such as those conducted by SAS2, COS-B and especially EGRET are very powerful at constraining the pulsar population. This was already appreciated by Harding (1981) who compared predictions of the polar cap model with the number of sources expected in the Galaxy. Bailes and Kniffen (1992) were the first to run a detailed pulsar population synthesis and compare $\gamma$-ray predictions with sky surveys. In Figure 5 we reproduce their realization of the Lyne, Manchester and Taylor (1985) pulsar population model for a polar cap $\gamma$-ray emission scheme compared with the galactic plane flux from SAS-2. Their work 

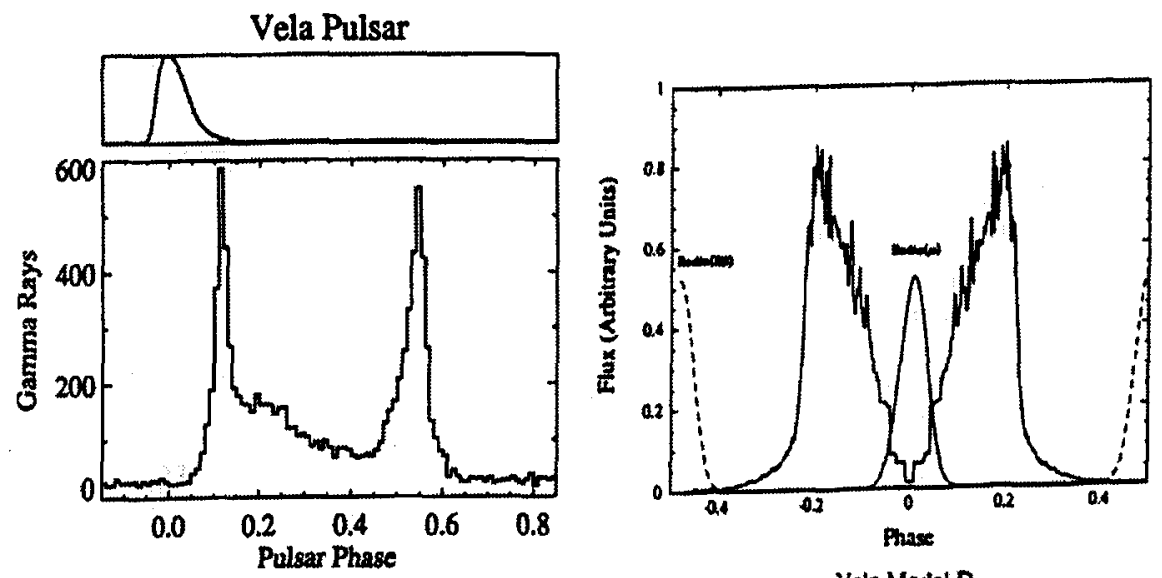

Vela Mocial D
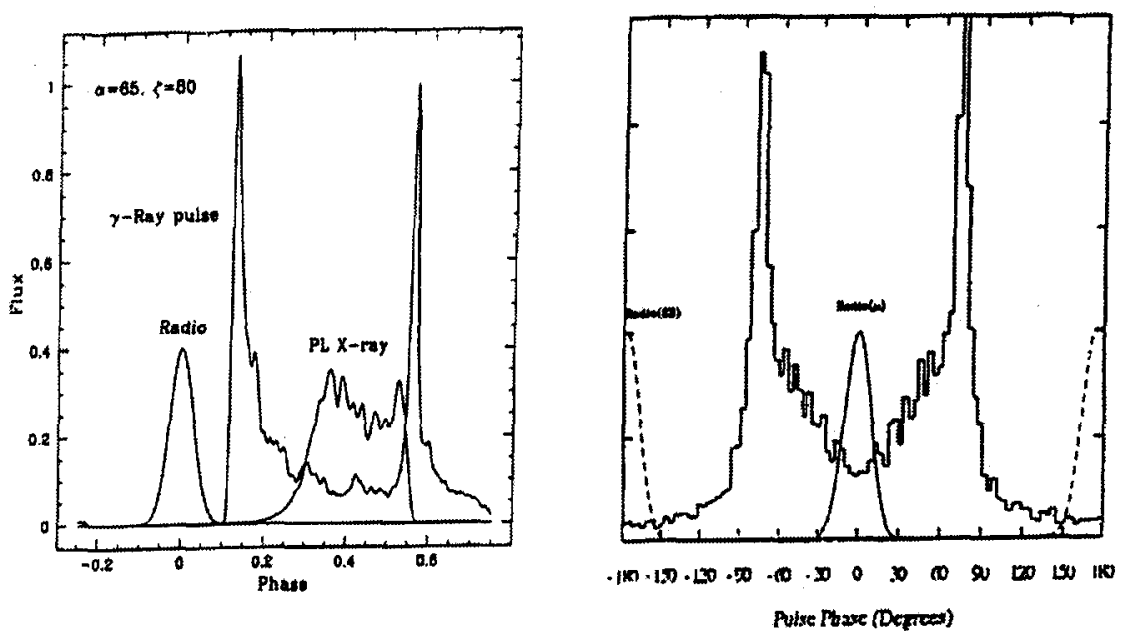

Figure 4. Vela pulse profile data and models. Clockwise from upper left: observed radio and $E>100 \mathrm{MeV}$ pulse profiles; DSM and DH models for polar cap $\gamma$-ray emission (with radio pulse shown at phase 0 , phase -0.5 is appropriate to 'exterior' models); and the outer magnetosphere Vela model of RY95 including the hard X-ray pulse from the base of the gap. 


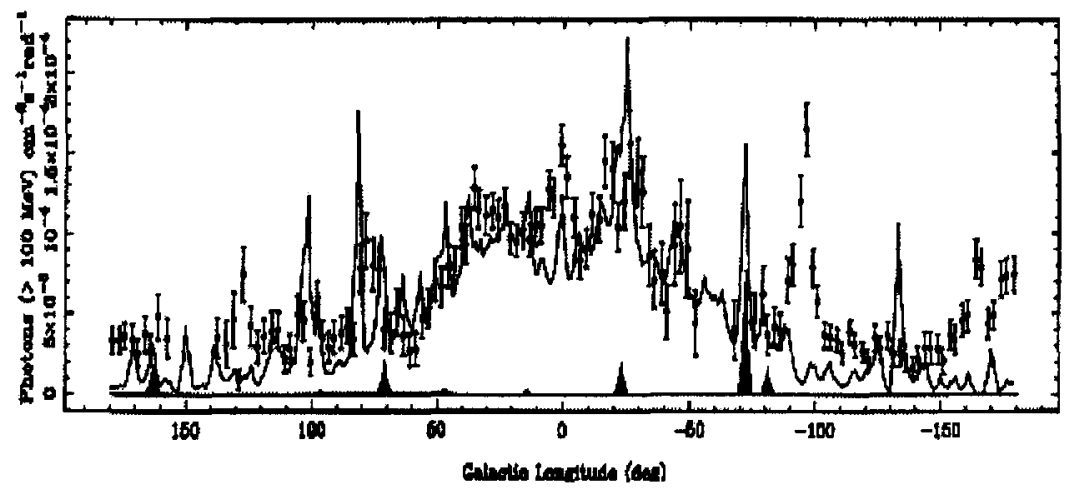

Figure 5. SAS-2 data points compared with Monte Carlo sampling of a $\gamma$-ray pulsar population based on the LMT model and a polar cap emission scheme (Bailes and Kniffen).

also showed that some constraint on the pulsar properties themselves could be extracted for a given $\gamma$-ray model. For example, the LMT model in which pulsars are born with $\langle\log B\rangle=11.9$ predicts a rather large total $\gamma$-ray flux; the model of Emmering and Chevalier (1991) with $\langle\log B\rangle=12.4$ in contrast produced fluxes in significantly better agreement with the data. This result follows from the $B$ dependence of total spin-down power for pulsars of a give age $\tau$, $\dot{E} \propto(B \tau)^{-2}$. Similar constraints were extracted by Schnepf $e t$ al. (1993) from COS-B data, who found, for example, that the polar cap picture of Harding (1981) would overpredict the COS-B flux for a pulsar birthrate $z 1 / 70 \mathrm{y}$.

In addition to the unresolved Galactic $\gamma$-ray flux, SAS2 and COS-B detected a number of Galactic point sources. Several authors noted that these seemed associated with regions of high mass star formation. Montmerle (1978) in particular described this association, calling the counterparts 'SNOB's (for SuperNova remnants and $\mathrm{OB}$ associations) and attributed the $\gamma$-ray flux to $\pi^{0}$ decay from interactions of SNR-generated cosmic rays. Inasfar as the point source population can also be identified with young pulsars, study of these sources can also constrain the physics of $\gamma$-ray emission.

The aforementioned population studies shared the assumption that the $\gamma$ ray radiation was isotropic and that all $\gamma$-ray pulsars would be seen in the radio. The discovery of the X-ray pulsations of Geminga (Halpern and Holt 1992) opened up the possibility that many of the unidentified $\gamma$-ray sources were indeed unobserved pulsars. Halpern and Ruderman (1993) and Helfand (1994) extrapolated from the case of Geminga to argue that pulsars not seen in the radio could indeed dominate the EGRET Galactic source catalog. In this way the case of Geminga has tied together the $\gamma$-ray pulse shape, beaming and population issues. In Yadigaroglu and Romani (1995) we ran a population synthesis for the outer magnetosphere picture, finding that the pulse shapes of known pulsars, the number of EGRET sources and the relative number of radio $+\gamma$ pulsars and 'Geminga's were well predicted. 


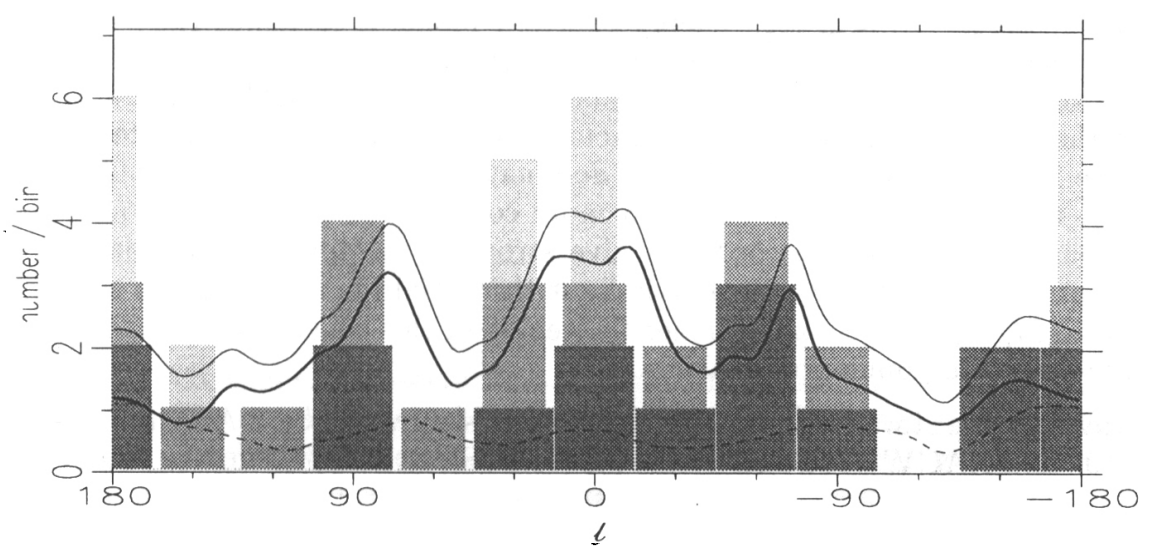

Figure 6. Longitude distribution of EGRET plane sources (Fierro 1996). Histogram bins (from dark to light) are pulsar IDsthigh confidence counterparts, superposition counterparts, and unassociated sources (largely AGN). Model curves give (bottom to top) predicted distribution of AGN, pulsars and their sum (YR96).

Efforts towards identification of these EGRET sources have made some progress. Sturner and Dermer (1995) showed that the sources were associated with SNR as a class, while Kaaret and Kottam (1996) have recently revisited Montmerle's association with $\mathrm{OB}$ clusters, showing that this connection is very strong. While definitive proof awaits pulse profile detections, the OB identifications and implied luminosities appear consistent with a large number of pulsars among the EGRET sources. Recently, we have identified individual 'counterparts' for many of these sources and compared the resulting positions and luminosity functions with predictions of pulsar models, including a detailed evolution model for outer magnetosphere $\gamma$-ray flux (Romani 1996). Modeling the Galactic distribution of high mass stars and the EGRET survey sensitivity in detail and synthesising the pulsar emission, Yadigaroglu and Romani (1996) find results for a pulsar birthrate of $1 / 100 \mathrm{y}$ reproduce the observed sources very well, in Galactic distribution (Figure 6), luminosity distribution and total number $(\sim 23)$. Comparison with polar cap models is useful; for example SD95's model of aligned pulsars with large ( $4 \times$ standard dipole) polar caps predicts 1.6 pulsars in the present catalog for the same birthrate and Galaxy model.

\section{Conclusions}

Pulse shape and population studies provide excellent opportunities for confronting pulsar emission models with the data. In this arena models placing the location of high energy $\gamma$-ray emission in the outer magnetosphere seem to fare quite well, but more work needs to be done. In particular, X-ray pulse data is proving quite difficult to interpret. Upcoming missions should, however provide very high signal-to-noise spectra for different components of the pulse. This holds great promise as a tool for understanding pulsar surface and magne- 
tospheric emissions. Once a high energy emission model is in hand, the $\gamma$-ray data in particular can teach us much about the pulsars themselves. For example YR96 substantiate the $\gamma$-ray constraints on the pulsar B field. They also find that the $\gamma$-ray pulsar sample, as extracted from the proposed candidates is largely free from the bias against high $n_{e}$ dominating the radio pulsar sample. In a sense, these sources provide a much more complete sample of the young pulsar population, and will accordingly give strong constraints on the conditions of neutron star birth. YR96 already limit the initial mass for neutron star formation to $M_{\mathrm{ZAMS}}>7 M_{\odot}$ and further tightening of this constraint will result from more complete population analysis.

Acknowledgments. Support for this work was provided by NASA grants NAG5-3101 and NAGW-4526 and by the Alfred P. Sloan foundation. Ion Yadigaroglu is thanked for assistance with modeling and preparation of the figures.

\section{References}

Bailes, M. and Kniffen, D.A. 1992, ApJ, 391, 659

Becker, W. 1996, in Proc. of 17th Texas Symposium, Böhringer, Morfil and Trümper, eds., in press

Chiang, J. and Romani, R.W. 1994, ApJ, 436, 754

Daugherty, J. and Harding, A.K. 1996, ApJ, in press (DH)

Emmering, R.T. and Chevalier, R.A. 1989, ApJ, 345, 931

Fierro, J.M. 1996, Doctoral Thesis, Stanford University

Fishman, G.J., Harnden, F.R. and Haymes, R.C. 1969, ApJ, 156, L107

Halpern, J.P. and Holt, S.S. 1992, Nature, 357, 222

Halpern, J.P. and Ruderman, M. 1993, ApJ, 415, 286

Harding, A.K. et al. 1981, ApJ, 245, 267

Helfand, D.J. 1994, MNRAS, 267, 490

Hillier, R.R. et al. 1970, ApJ, 162, L177

Kaaret, P. and J. Cottam 1996, ApJ, in press

Lyne, A., Manchester, R. and Taylor, J.H. 1985, MNRAS, 213, 613

Montmerle, T. 1979, ApJ, 231, 95

Ögelman, H. 1995 in Lives of Neutron Stars, eds. J. van Paradijs \& A. Alpar (Dordrecht: Kluwer), 101

Romani, R.W. 1996, ApJ, in press (R96)

Romani, R.W. and Yadigaroglu, I.-A. 1995, ApJ, 438, 314 (RY95)

Schnepf, N.G. et al. 1993, AIP Conf. Proc., 280, 85

Sturner, S.J. Dermer, C.D. and Michel, F.C. 1995, ApJ, 445, 736

Sturner, S.J. and Dermer, C.D. 1995, A\&A, 293, L17

Thompson, D.J. 1975, ApJ, 201, L117

Thompson, D.J., et al. 1994, ApJ, 436, 229

Yadigaroglu, I.-A. and R.W. Romani 1995, ApJ, 449, 211 (YR)

Yadigaroglu, I.-A. and R.W. Romani 1995, ApJ, submitted (YR96) 